\title{
COMPOSICIÓN FLORÍSTICA Y ESTRUCTURA DE LA VEGETACIÓN SECUNDARIA en EL NORTE DEL ESTADO DE CAMPECHE, MÉXICO
}

\author{
Pedro Zamora-Crescencio ${ }^{1,4}$, María del Rosario Domínguez-Carrasco ${ }^{1}$, Pascale Villegas ${ }^{1}$, \\ Celso Gutiérrez-Báez ${ }^{1}$, luis Ariel Manzanero-Acevedo², Juan José Ortega-Haas ${ }^{3}$, \\ Saraí Hernández-Mundo 3 , Elda Celedonia Puc-Garrido ${ }^{3}$ y Rosalía Puch-Chávez ${ }^{3}$ \\ ${ }^{1}$ Centro de Investigaciones Históricas y Sociales, Universidad Autónoma de Campeche \\ ${ }^{2}$ Centro de Estudios en Desarrollo Sustentable y Aprovechamiento de la Vida Silvestre, \\ Universidad Autónoma de Campeche \\ ${ }^{3}$ Facultad de Ciencias Químico Biológicas, Universidad Autónoma de Campeche \\ ${ }^{4}$ Autor para la correspondencia: yecver@hotmail.com
}

\begin{abstract}
Resumen: Se estudió la composición y la estructura de la vegetación secundaria de diez años de edad en el área de Tepakán, Campeche, México. Se delimitaron 20 parcelas de $5 \times 10 \mathrm{~m}^{2}\left(1,000 \mathrm{~m}^{2}\right.$ en total $)$, midiendo todos los individuos de $\geq 2 \mathrm{~cm}$ de DAP. El análisis estructural de la vegetación se basó en los valores de importancia de las especies. La diversidad se estimó con el índice de Shannon-Wiener (H'). Se encontraron 1,150 individuos leñosos de 43 especies, agrupadas en 35 géneros y 21 familias. El valor de H' fue de 1.59. La familia con mayor número de especies fue Fabaceae (14 especies). Cordia alliodora fue la más importante con base en su elevado valor de densidad y dominancia relativa, seguida en importancia por Piscidia piscipula y Acacia gaumeri, las que aglutinaron $33.9 \%$ del total del valor de importancia. Estas especies son las más importantes en la estructura y fisonomía de la comunidad vegetal.
\end{abstract}

Palabras claves: composición, estructura, florística, Tepakán, vegetación secundaria.

\begin{abstract}
We studied species composition and structure of a ten year-old secondary forest in Tepakán, Campeche, Mexico. We marked 20 plots of $5 \times 10 \mathrm{~m}^{2}\left(1,000 \mathrm{~m}^{2}\right.$ in total), where all individuals with a DBH $\geq 2 \mathrm{~cm}$ were measured. Vegetation structure was characterized using the importance values of the species, and species diversity was estimated with the Shannon-Wiener (H') Index. We recorded 1,150 woody individuals in 43 species, 35 genera, and 21 families. Diversity was H'= 1.59. The Fabaceae was the family with the highest number of species (14). Cordia alliodora was the most important species according to its large stem density, followed by Piscidia piscipula and Acacia gaumeri. Together they account for $33.9 \%$ of total importance value. These species are the most important in the structure and physiognomy of the community.
\end{abstract}

Key words: composition, floristic, secondary vegetation, structure, Tepakán.

$\mathbf{L}$ as selvas tropicales son un recurso natural importante, ya que regulan la atmósfera a nivel global, proporcionan hábitats para numerosas especies vegetales y animales, regulan el sistema hidrológico a nivel local y regional y con el reciclaje de sus nutrimentos permiten la fertilidad natural del suelo. Además brindan a las poblaciones rurales y urbanas, maderas útiles en la construcción de viviendas, forraje, plantas alimenticias, combustible y medicinales, entre otras (Granielo-Vásquez, 1985; Sánchez-Aguilar y Rebollar-Domínguez, 1999; Martínez-Ramos y García-Orth, 2007).
La demanda del recurso vegetal para la industria y el sustento de la población humana en las regiones con selvas tropicales han causado la pérdida, además de una fuerte conversión de estas comunidades vegetales en áreas agrícolas, pecuarias y asentamientos humanos (Gehring et al., 1999; Arias-Escobar y Barrera-Cataño, 2007). Como resultado se han perdido dos terceras partes de las selvas tropicales del planeta (Wilson, 1988). Este cambio en el uso del suelo ha provocado cambios en la estructura y composición florística de los remanentes de selva y el confinamiento de la ve- 
getación primaria a pequeños manchones y la creación de grandes áreas de vegetación secundaria (Ramírez-Marcial et al., 1998; Trejo y Dirzo, 2000; Orians, 2000; Guariguata y Ostertag, 2002; Herrerías-Diego y Benítez-Malvido, 2005; Montenegro-S. et al., 2006; González-Castillo et al., 2007). Estas áreas están rápidamente incrementando en extensión (Chazdon y Coe, 1999; Chazdon et al., 2010).

La vegetación secundaria es una comunidad compuesta por una composición florística variable en función del tiempo de abandono (Giraldo-Cañas, 2000; Castillo-Campos y Laborde-D, 2004), que se manifiesta después de que una selva tropical primaria ha sido perturbada por factores como: incendios naturales, caída de árboles por vientos fuertes, extracción selectiva de árboles, actividad agropecuaria, entre otros (Gómez-Pompa y Vázquez-Yanes, 1985).

La vegetación primaria de la Península de Yucatán ha sido transformada y sustituida por diferentes usos de la tierra, fuego y huracanes (Allen et al., 2003). Los pocos sitios donde existe vegetación madura muestra signos de perturbación humana (Rico-Gray y García-Franco, 1992) y casi en su totalidad, la Península es vegetación secundaria (Rico-Gray y García-Franco, 1991). Estos cambios han provocado modificaciones en la estructura de la vegetación, composición florística (Carnevali et al., 2003), diversidad, abundancia y frecuencia de las especies (Ramírez-Marcial et al., 1998; Sánchez-Aguilar y Rebollar-Domínguez, 1999, Von-Gadow et al., 2004).

Estudios realizados sobre vegetación secundaria de bosques húmedos y selvas secas tropicales han documentado cambios en su estructura y composición florística (Lott et al., 1987; Guariguata et al., 1997; Gehring et al., 1999; Giraldo-Cañas, 2000, 2001; Guariguata y Ostertag, 2001; Balvanera et al., 2002; Peña-Claros, 2003; Segura et al., 2003; Ruprecht, 2005; Sagar y Singh, 2005; Balvanera y Aguirre, 2006; Toledo y Salick, 2006; Durán et al., 2006; Lebrija-Trejos et al., 2008; Chazdon et al., 2010). Desafortunadamente, el conocimiento básico de la estructura y la diversidad de especies leñosas de la vegetación secundaria en la Península de Yucatán, han recibido poca atención (Rico-Gray y García-Franco, 1992; Mizrahi et al., 1997; González-Iturbe et al., 2002). En Campeche existen trabajos en los que se aporta información cualitativa sobre vegetación y flora (Rico-Gray, 1982; Flores y Espejel, 1994; ZamoraCrescencio, 1999, 2003) y algunos estudios cuantitativos de la vegetación (Durán-García, 1987, 1995; Martínez y Galindo-Leal, 2002; Díaz-Gallegos et al., 2002). Sin embargo, no hay estudios que evalúen aspectos cuantitativos sobre la estructura de la vegetación secundaria derivada de la selva baja caducifolia.

Para la Península de Yucatán la selva baja caducifolia se caracteriza porque los árboles que la conforman (75\% o más) pierden las hojas en tiempo de sequía; por la abundancia de leguminosas y por las especies endémicas (Flores y Espejel, 1994). La altura de sus árboles oscila entre seis y 12 metros (Miranda y Hernández-X., 1963) y sus diámetros entre diez y $30 \mathrm{~cm}$ (Flores y Espejel, 1994). Las especies que caracterizan a la selva baja caducifolia son: Acacia gaumeri, Alvaradoa amorphoides, Bursera simaruba, Caesalpinia gaumeri, C. yucatanensis, Ceiba aesculifolia, Diospyros cuneata, Gymnopodium floribundum, Hampea trilobata, Jatropha gaumeri, Neomillspaughia emarginata, Parmentiera aculeata, Piscidia piscipula, Randia longiloba, Sideroxylon americanum, entre otras (Miranda, 1978; Flores y Espejel, 1994). Para la parte norte del estado de Campeche (zona de estudio), esta comunidad vegetal ha sido afectada por las actividades humanas principalmente para cultivar maíz, y extracción de algunas especies como el kitimche' (Caesalpinia gaumeri) usado como horcones de casas y el sak iitsa' (Neomillspaughia emarginata) para mangos de escobas (com. pers. Gilberto Cobo), en donde prácticamente la selva baja caducifolia se encuentra en fases secundarias de regeneración. El objetivo de este trabajo fue proporcionar información básica sobre la estructura, la composición florística y la diversidad de una etapa sucesional con diez años de abandono en el norte del estado de Campeche.

Sitio de estudio. El sitio de estudio se localiza a $2 \mathrm{~km}$ al este de la carretera federal Campeche-Mérida, en el ejido de Tepakán, municipio de Calkini, estado de Campeche (20 $24^{\circ}$ 00 ” N y $90^{\circ} 01$ ' 00" O, Figura 1). La topografía del terreno es básicamente plana, con algunos lomeríos suaves no muy prominentes. Las rocas son sedimentarias del Cenozoico y los afloramientos en extensión son rocas calizas, margas, lutitas y areniscas de edad Eocénica del Terciario Inferior. Entre las calizas se encuentran sedimentos calcáreos de aspecto crestoso, microcristalinos, pulverulentos o sacaroides de color blanco (Flores y Espejel, 1994; INEGI, 1985a). El

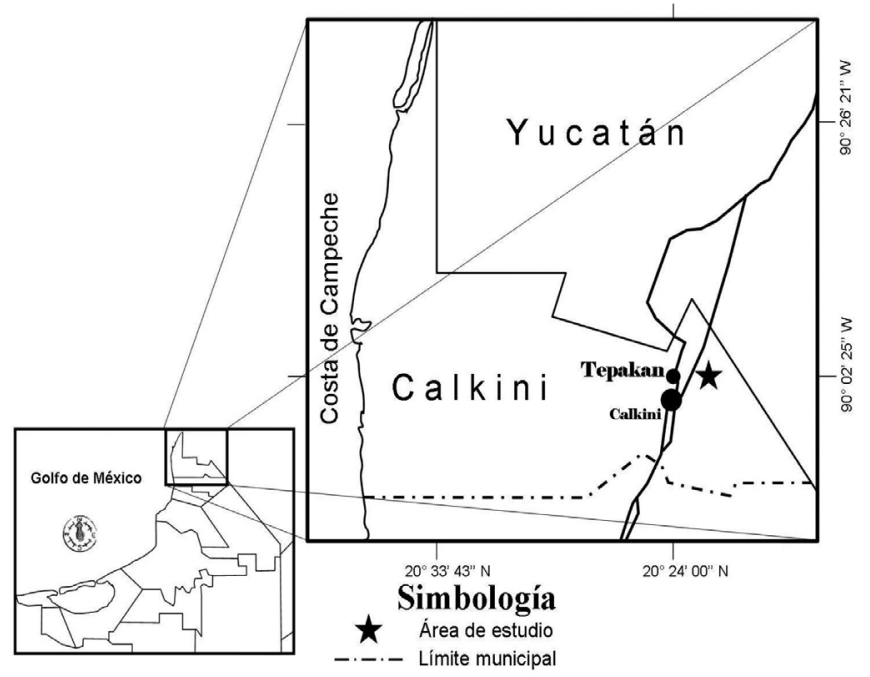

Figura 1. Localización del área de estudio. 
suelo es de tipo luvisol combinado con litosol, presenta un horizonte ócrico, sin reacción al $\mathrm{HCl}$; la estructura es granular de tamaño fino y con moderado desarrollo. Este tipo de suelo es de fertilidad moderada, de textura fina arcillosa, color rojo o pardo rojizo (INEGI, 1985b). El clima se clasifica como Awo, cálido subhúmedo con régimen de lluvias en verano. La temperatura media anual es de $25.8^{\circ} \mathrm{C}$ y la precipitación total anual es $1,229.4 \mathrm{~mm}$; marzo es el mes de mínima precipitación y septiembre tiene los valores más altos (CONAGUA, 2007).

Método. El sitio de muestreo se seleccionó al azar y, con la ayuda de un guía local, se determinó que se trataba de una comunidad con diez años de abandono, derivada de selva baja caducifolia. El muestreo de la vegetación se realizó en dos periodos: mayo del $2008\left(750 \mathrm{~m}^{2}\right)$ y en junio del 2010 $\left(250 \mathrm{~m}^{2}\right)$, cubriendo una superficie de $1,000 \mathrm{~m}^{2}(0.1 \mathrm{ha})$, usando parcelas de $5 \times 10 \mathrm{~m}\left(50 \mathrm{~m}^{2}\right)$, dando un total de 20 parcelas. En cada parcela se midieron todos los árboles y arbustos con diámetro a la altura del pecho (DAP) $\geq 2 \mathrm{~cm}$. Para la identificación taxonómica de las especies se recolectaron especímenes tanto fértiles como estériles para ser cotejados con los ejemplares de la colección del herbario UCAM del Centro de Investigaciones Históricas y Sociales de la Universidad Autónoma de Campeche.

Análisis de los datos. La estructura de la vegetación se analizó en términos de área basal, usando la distribución de frecuencias agrupadas por clases diamétricas. La estructura vertical se analizó usando un histograma de frecuencias de alturas para todos los individuos censados. Las distintas clases de diámetro y altura fueron definidas considerando los valores de los datos y el número total de individuos. Para determinar el valor de importancia de las especies, se calcularon y sumaron la densidad relativa (número de individuos por especie/total de individuos de las especies $\times 100$ ), la frecuencia relativa (número de unidades de muestra en el que se encontró una especie/el número total de unidades de muestra $\times 100)$ y la dominancia relativa (área basal de cada especie/total del área basal de todas las especies $\times 100$ ) (Mueller-Dombois y Ellenberg, 2002). La diversidad de especies se estimó usando el índice de Shannon-Wiener $\left(\mathrm{H}^{\prime}\right)$ (Magurran, 1988):

$\mathrm{H}^{\prime}=-(\mathrm{pi})(\ln \mathrm{pi})$

donde pi = proporción de la muestra que corresponde a la especie i

La descripción de la composición y estructura se realiza agrupando los resultados de los dos periodos debido a que no fueron parcelas permanentes.

Composición florística. Se registraron 43 especies leñosas en 35 géneros y 21 familias en 0.1 ha (Cuadro 1, Apéndi-
Cuadro 1. Características de las especies leñosas en dos periodos de muestreo.

\begin{tabular}{lccc}
\hline Variables & $\begin{array}{c}\text { Periodo } \\
2008\end{array}$ & $\begin{array}{c}\text { Periodo } \\
2010\end{array}$ & Total en los dos periodos \\
\hline Área muestreada & $750 \mathrm{~m}^{2}$ & $250 \mathrm{~m}^{2}$ & $1000 \mathrm{~m}^{2}(0.1 \mathrm{ha})$ \\
Número de individuos & 837 & 313 & 1,150 \\
Familias & 21 & 11 & 21 \\
Géneros & 36 & 17 & 35 \\
Especies & 42 & 21 & 43 \\
Índice Shannon-Weaner & 1.29 & 0.55 & 1.59 \\
Equitatividad & 0.79 & 0.34 & 0.98 \\
Área basal (m²/ha) & 14.87 & 27.43 & 18.0 \\
\hline
\end{tabular}

ce). Con base en las 20 parcelas de muestreo, las familias con mayor riqueza de especies fueron Fabaceae (14) y Ebenaceae (3), que concentraron $39.53 \%$ del total de las especies encontradas. Las especies con las mayores densidades fueron Acacia gaumeri (179 individuos), Cordia alliodora (177), Lonchocarpus xuul (107), Piscidia piscipula (87), Sideroxylon obtusifolium (86) y Lysiloma latisiliquum (54). Éstas representan $60.09 \%$ del total de los individuos registrados. Entre las especies raras (aquellas con menos de 50 individuos) estuvieron Sideroxylon americanum (47 individuos), Acacia sp. (36), Diospyros anisandra (34), Hippocratea excelsa (34), Colubrina sp. (32) y Caesalpinia gaumeri (27). La diversidad y equitatividad fueron de 1.59 y 0.98 , respectivamente, valores que indican que la comunidad vegetal tiene una diversidad baja.

Análisis estructural. En el área de $1,000 \mathrm{~m}^{2}$ se registraron 1,150 individuos con DAP $\geq$ de $2 \mathrm{~cm}$ (Cuadro 1). La densidad de individuos de dos a $3.9 \mathrm{~cm}$ fue de $8,280 \mathrm{ind} / \mathrm{ha}$ (70\%), mientras que la densidad de $2,940 \mathrm{ind} / \mathrm{ha}$ corresponde a las clases dos, tres y cuatro $(25.57 \%)$ y en el resto de las clases la densidad fue de 280 ind/ha (2.4\%) (Figura 2). Acacia gaumeri fue la que tuvo mayor densidad (1,790 ind/ ha), seguida de Cordia alliodora (1,770 ind/ha) y Lonchocarpus xuul (1,070 ind/ha), mientras que Piscidia piscipula y Sideroxylon obtusifolium tuvieron bajas densidades $(870$ ind/ha). Estas cinco especies constituyen el 55.5\% (637) del total de individuos, el resto se distribuye entre 38 especies.

En la figura 2 se muestra que la distribución de los individuos en las diferentes clases diamétricas presenta la forma característica de una J-invertida, lo que indica que la mayoría de los individuos están en la clase de tamaño pequeño. Las especies que aportaron mayor número (> 100) de individuos en las dos primeras clases diamétricas fueron Acacia gaumeri (168 individuos), Cordia alliodora (163) y Lonchocarpus xuul (106), mientras que las especies que alcanzaron la última clase diamétrica $(>16 \mathrm{~cm}$ ) fueron Caesalpinia gaumeri, Havardia albicans, Lysiloma latisiliquum, Piscidia piscipula y las que aportaron más indivi- 


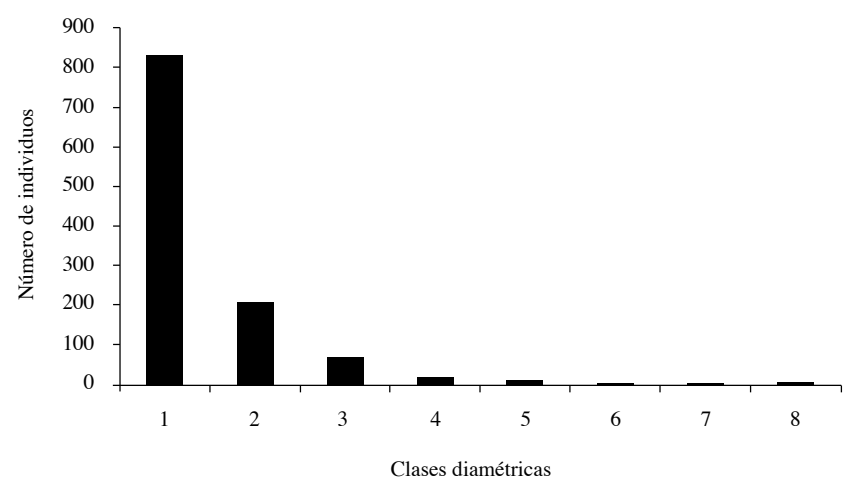

Figura 2. Distribución de las clases diamétricas de los individuos con DAP $\geq 2.0 \mathrm{~cm}$ de las 20 parcelas de muestreo de la vegetación secundaria de diez años de abandono en Tepakán, Campeche, México. Clases de diámetros: $1=2-3.9 \mathrm{~cm} ; 2$ = 4-5.9 $\mathrm{cm} ; 3=6-7.9 \mathrm{~cm} ; 4=8-9.9 \mathrm{~cm} ; 5=10-11.9 \mathrm{~cm} ; 6=12-13.9 \mathrm{~cm}$;

$$
7=14-15.9 \mathrm{~cm} ; 8=>16 \mathrm{~cm} \text {. }
$$

duos en esta clase fueron Lysiloma latisiliquum y Piscidia piscipula.

En relación con la distribución vertical por clases de altura, el mayor número de individuos (511) se concentró en la segunda clase (2.6-3.5 m), y se muestra un decremento progresivo hacia las tres últimas clases (Figura 3). Las especies representativas que tuvieron mayor proporción de individuos en esta clase (2.6-3.5 m) de altura fueron Acacia gaumeri (103 individuos), Cordia alliodora (73), Sideroxylon obtusifolium (43), Lonchocarpus xuul (37) y Lysiloma latisiliquum (33). Cordia alliodora fue una especie común en las primeras tres clases de altura. La última clase (5.6$6.5 \mathrm{~m}$ ) la componen individuos de las especies Acacia sp., Caesalpinia gaumeri, Cordia alliodora, Havardia albicans y Piscidia piscipula.

En el apéndice se señalan los componentes y el valor de

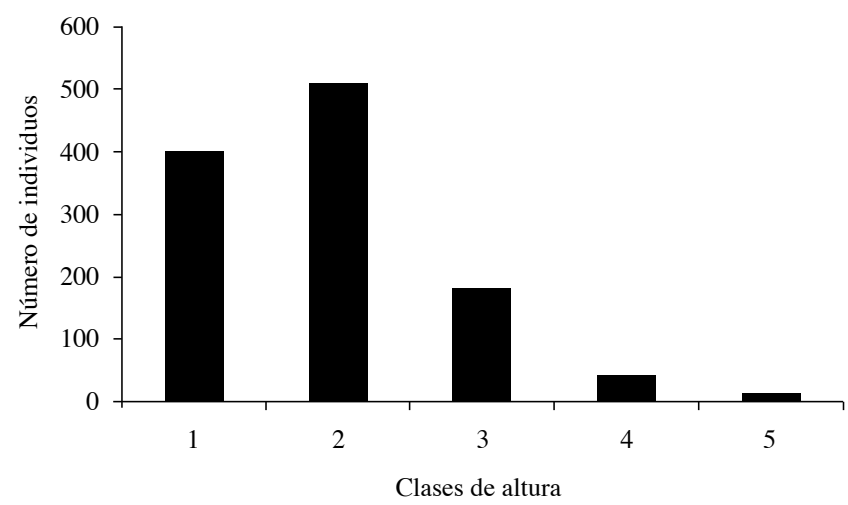

Figura 3. Distribución de las clases de altura de los individuos con DAP $\geq 2.0 \mathrm{~m}$ de las 20 parcelas de vegetación secundaria con diez años de abandono en Tepalkan, Campeche, México. Clases de altura: $1=2-2.5 \mathrm{~cm} ; 2=2.6-3.5 \mathrm{~cm} ; 3=3.6-4.5 \mathrm{~cm} ; 4=4.6-5.5$ $\mathrm{cm} ; 5=5.6-6.5 \mathrm{~cm}$. importancia de las especies que conforman la estructura de la vegetación secundaria. Entre las especies más importantes se encuentran Acacia gaumeri, Cordia alliodora y Piscidia piscipula que concentran $33.9 \%$ del total del valor de importancia relativa y que son los componentes florísticos más sobresalientes de esta comunidad vegetal. Cordia alliodora fue la especie más importante por su valor de importancia en la composición de la comunidad, destacando por su alta densidad relativa más que por su área basal. Acacia gaumeri ocupó el tercer lugar, a pesar de ocupar el segundo lugar en densidad relativa; esto se debe a que sus áreas basales fueron bajas, mientras que Piscidia piscipula ocupó el segundo lugar, su posición está determinado por el área basal.

Los resultados muestran que la riqueza específica (43 especies) difiere cuando se la compara con otras comunidades sucesionales [Rico-Gray et al., 1988 (54 especies); Mizrahi et al., 1997 (41 especies); González-Iturbe et al., 2002 (66 especies), Ceccon et al., 2002 (33 especies)]. Es posible que las diferencias se deban al tipo de vegetación original, al tamaño de la parcela, el uso al que estuvo dedicada antes del abandono y a la aplicación de criterios del tamaño de los diámetros utilizados. Por otra parte, las condiciones topográficas y geológicas son similares, a excepción de las comunidades estudiadas por Rico-Gray et al. (1988), que derivan de una selva mediana subcaducifolia y con un poco más de humedad en esos sitios, que en la parte norte de la propia Península.

La familia Fabaceae fue la que tuvo mayor número de especies, lo cual coincide con los reportes de muchos otros estudios florísticos realizados de selva baja caducifolia (Lott et al., 1987; Gentry, 1995; Gillespie et al., 2000; GallardoCruz et al., 2005; Durán et al., 2006; Pineda-García et al., 2007; Alanís-Rodríguez et al., 2010) y, particularmente, de la península de Yucatán (Ceccon et al., 2002; González-Iturbe et al., 2002). La familia Fabaceae es la mejor representada debido a que es una de las más diversas en el mundo y ampliamente distribuida en las regiones tropicales (Rzedowski, 1978; Gentry, 1988; Sousa-S. y Delgado-S., 1998; Lavin et al., 2005). Si comparamos las distintas especies que han sido reportadas en éste y otros estudios con el mismo tipo de hábitat (Flores, 1989; Mizrahi et al., 1997; Ceccon et al., 2002; González-Iturbe et al., 2002), encontramos que hay tres especies comunes: Havardia albicans, Mimosa bahamensis y Piscidia piscipula. Estas son las especies características desde las primeras etapas hasta las fases más avanzadas de regeneración natural (Flores, 1989; Mizrahi et al., 1997; González-Iturbe et al., 2002).

Por otro lado, las densidades encontradas para Acacia gaumeri y Cordia alliodora, coinciden con los reportes de las comunidades secundarias estudiadas por Marín-Chávez (1997) y Mizrahi et al. (1997). Es posible aventurar dos posi- 
bles explicaciones para esto, una que son especies de rápido crecimiento (Flores, 1989; Mizrahi et al., 1997; GonzálezIturbe et al., 2002) y la otra podría ser su adaptación a condiciones de disturbio por actividades humanas en el pasado (Rzedowski, 1978; Thompson et al., 2002). Con respecto a las áreas basales, Cordia alliodora, Lysiloma latisiliquum y Piscidia piscipula presentaron densidades abundantes en las primeras clases diamétricas, lo cual es un ejemplo de regeneración de estas especies en la comunidad. Las tres especies tienen alta capacidad de rebrote después de una perturbación y son de rápido crecimiento (Mizrahi et al., 1997; Durán et al., 2002; González-Iturbe et al., 2002). Por otra parte, sólo tres especies (Cordia alliodora, Lonchocarpus xuul y Sideroxylon obtusifolium) estuvieron representadas en las cuatro clases diamétricas con una mayor densidad, en particular la primera, lo cual sugiere que forman parte importante de la composición y estructura de la vegetación secundaria en el área de estudio. Es probable, que estas especies tengan la capacidad de sobrevivir a medida que se incrementa la competencia entre especies, y que su establecimiento sea favorecido por la perturbación frecuente donde se ha desmontado y la acción del hombre ha sido persistente (Collins et al., 1995; Martínez y Galindo-Leal, 2002; Pennington y Sarukhán, 2005). Los individuos de altura $(6 \mathrm{~m})$ y diámetro $(39 \mathrm{~cm})$ mayor corresponden a especies como Caesalpinia gaumeri, Cordia alliodora, Havardia albicans y Piscidia piscipula, su presencia posiblemente se deba a que fueron toleradas al tumbar la vegetación para fines agrícolas.

Las familias Boraginaceae y Fabaceae fueron estructuralmente dominantes en la comunidad sucesional estudiada, lo cual coincide con otros trabajos (Flores, 1989; Mizrahi et al., 1997; Ceccon et al., 2002; González-Iturbe et al., 2002), pero su importancia parece disminuir en comunidades sucesionales más avanzadas (Rico-Gray et al., 1988).

El índice de diversidad encontrada en este estudio ( $\mathrm{H}^{\prime}$ $1.59,43 \mathrm{spp}$.) comparado con otros estados sucesionales secundarios en Yucatán muestra que la diversidad fue ligeramente alta [Ceccon et al. (2002) (H' 1.23, 33 spp.), Mizrahi et al. (1997) (H'1.16, 41 spp.) y de González-Iturbe et al. (2002) (H' 1.39, 66 spp.)]. Estas diferencias podrían estar relacionadas con la abundancia y riqueza de especies, con las condiciones ecológicas del hábitat y con los cambio en el uso del suelo de cada sitio de estudio (Segura et al., 2003; Alanís-Rodríguez et al., 2008).

Se agradece a los doctores Victor Rico-Gray, William J. Folan H. y Jorge Meave del Castillo por la revisión del manuscrito y el resumen en inglés. Las sugerencias de cambios que hicieron dos árbitros anónimos para una mejor versión del manuscrito. Al Lic. Juan José Cosgaya Medina por la elaboración de la figura 1, al Sr. Gilberto Cobo, guía local y a la Biól. Gisela Aguayo Dionet por el apoyo de campo. Este trabajo fue apoyado por el proyecto "Cultivo de especies de uso artesanal en Tepakán, Calkiní, Campeche" financiado por Fundación Produce 2005 (Clave 1337) y el proyecto FOMIX CAMPECHE-CONACYT (Clave CAMP-200893419) en la segunda salida de campo.

Alanís-Rodríguez E., Jiménez-Pérez J., Aguirre-Calderón O., Treviño-Garza E, Jurado-Ybarra E. y González-Tagle M. 2008. Efecto del uso del suelo en la fitodiversidad del matorral espinoso Tamaulipeco. Ciencia UANL :56-62.

Alanís-Rodríguez E., Aranda-Ramos R., Mata-Balderas J.M., Canizales-Velázquez P.A., Jiménez-Pérez J., Uvalle-Sauceda J.I., Valdecantos-Dema A. y Ruiz-Bautista M.G. 2010. Riqueza y diversidad de especies leñosas del bosque tropical caducifolio en San Luis Potosí, México. Ciencia UANL :287-293.

Allen E.B., Allen M.F., Violi H.A. y Gómez-Pompa A. 2003. Restoration of tropical seasonal forest in Quintana Roo. En: Gómez-Pompa A., Allen M.F., Fedick S.L. y Jiménez-Osornio J.J. Eds. The Lowland Maya Area: Three Millennia at the HumanWildland Interface, pp. 587-598, Food Products, Nueva York.

Arias-Escobar M.A. y Barrera-Cataño J.I. 2007. Caracterización florística y estructural de la vegetación vascular en áreas con diferente condición de abandono en la cantera soratama, localidad de Usaquén, Bogotá. Universitas Scientiarum. Edición especial II, :25-45.

Balvanera P., Lott E., Segura G., Siebe C. y Islas A. 2002. Patterns of -diversity in a Mexican tropical dry forest. Journal of Vegetation Science $: 145-158$.

Balvanera P. y Aguirre E. 2006. Tree diversity, environmental heterogeneity, and productivity in a Mexican tropical dry forest. Biotropica :479-491.

Carnevali G., Ramírez I.M. y González-Iturbe J.A. 2003. Flora y vegetación de la Península de Yucatán. En: Colunga-GarcíaMarín P. y Larqué-Saavedra A. Eds. Naturaleza y Sociedad en el Área Maya, Pasado, Presente y Futuro, pp. 53-68, Academia Mexicana de Ciencias y Centro de Investigación Científica de Yucatán, México, D. F.

Castillo-Campos G. y Laborde-D J. 2004. La vegetación. En: Guevara-S S., Laborde-D J. y Sánchez-Ríos G. Eds. Los Tuxtlas. El Paisaje de la Sierra, pp. 231-265, Instituto de Ecología, A.C. y Unión Europea, Xalapa.

Ceccon E., Olmsted I., Vázquez-Yanes C. y Campos-Alves J. 2002. Vegetation and soil properties in two tropical dry forests of differing regeneration status in Yucatan. Agrociencia:621631.

Chazdon R.L. y Coe F.G. 1999. Ethnobotany of woody species in second growth, old-growth, and selectively logged forests of Northeastern Costa Rica. Conservation Biology :1312-1322.

Chazdon R.L., Finegan B., Capers R.S., Salgado-Negret B., Casanoves F., Boukili V. y Norden N. 2010. Composition and dynamics of functional groups of trees during tropical forest succession in northeastern Costa Rica. Biotropica :31-40.

CONAGUA [Comisión Nacional del Agua] 2007. Base de datos mensuales climatológicos correspondientes a la estación de Calkiní, Camp. México. Residencia Técnica Jefatura de Aguas Superficiales. Organización de Cuenca Península de Yucatán, 
Mérida.

Collins S.L., Glenn S.M. y Gibson D.J. 1995. Experimental analysis of intermediate disturbance and initial floristic composition decoupling cause and effect. Ecology :486-492.

Díaz-Gallegos J.R., Castillo-Acosta O. y García-Gil G. 2002. Distribución espacial y estructura arbórea de la selva baja subperennifolia en un ejido de la Reserva de la Biosfera Calakmul, Campeche, México. Universidad y Ciencia :11-28.

Durán-García R. 1987. Descripción y análisis de la estructura y composición de la vegetación de los petenes del noroeste de Campeche, México. Biótica:181-198.

Durán-García R. 1995. Diversidad florística de los petenes de Campeche. Acta Botanica Mexicana :73-84.

Durán E., Balvanera P., Lott E., Segura G., Pérez-Jiménez A., Islas A. y Franco M. 2002. Estructura, composición y dinámica de la vegetación. En: Noguera F.A., Vega-Rivera J.H., GarcíaAldrete A.N. y Quesada-Avendaño M. Eds. Historia Natural de Chamela, pp. 443-472, Universidad Nacional Autónoma de México, Instituto de Biología, México, D.F.

Durán E., Meave J.A., Lott E.J. y Segura G. 2006. Structure and tree diversity patterns at the landscape level in a mexican tropical deciduous forest. Boletín de la Sociedad Botánica de México :43-60.

Flores J.S. 1989. Importancia de las leguminosas en la vegetación secundaria del Estado de Yucatán. Revista Universidad Autónoma de Yucatán :68-80.

Flores J.S. y Espejel I. 1994. Tipos de vegetación de la Península de Yucatán. Etnoflora Yucatanense, Fascículo 3. Universidad Autónoma de Yucatán, Mérida.

Gallardo-Cruz J.A., Meave J.A. y Pérez-García E.A. 2005. Estructura, composición y diversidad de la selva baja caducifolia del Cerro Verde, Nizanda (Oaxaca), México. Boletín de la Sociedad Botánica de México :19-35.

Gehring C., Denich M., Kanashiro M. y Vlek P.L.G. 1999. Response of secondary vegetation in eastern Amazonia to relaxed nutrient availability constraints. Biogeochemistry :223-241.

Gentry A.H. 1988. Changes in plant community diversity and floristic composition on environmental and geographical gradients. Annals of the Missouri Botanical Garden :1-34.

Gentry A.H. 1995. Diversity and floristic composition of neotropical dry forest. En: Bullock S.H., Mooney H.A. y Medina E. Eds. Seasonally Dry Tropical Forest, pp. 146-190, Cambridge University Press, Cambridge.

Gillespie T.W., Grijalva A. y Farris C.N. 2000. Diversity, composition, and structure of tropical dry forest in Central America. Plant Ecology:37-47.

Giraldo-Cañas D. 2000. Variación de la diversidad florística en un mosaico sucesional en la cordillera central andina (Antioquia, Colombia). Darwiniana :33-42.

Giraldo-Cañas D. 2001. Análisis florístico y fitogeográfico de un bosque secundario pluvial andino, cordillera central (Antioquia, Colombia). Darwiniana:187-199.

Gómez-Pompa A. y Vázquez-Yanes C. 1985. Estudios sobre la regeneración de selvas en regiones cálido-húmedas de México. En: Gómez-Pompa A y del Amo S. Eds. Investigaciones sobre la Regeneración de Selvas Altas en Veracruz, México, Vol. II, pp 1-25, Instituto Nacional de Investigaciones sobre los Recursos Bioticos y Editorial Alhambra Mexicana, México, D.F.

González-Castillo O., Jiménez-de Azcárate J., García-Pérez J. y Aguirre-Rivera J.R. 2007. Flórula vascular de la Sierra de Ca- torce y territorios adyacentes, San Luis Potosí, México. Acta Botanica Mexicana :1-38.

González-Iturbe J.A., Olmsted I. y Tun-Dzul F. 2002. Tropical dry forest recovery after long term Henequen (sisal, Agave fourcroydes Lem.) plantation in northern Yucatan, Mexico. Forest Ecology and Management :67-82.

Granielo-Vásquez S. 1985. Uso y abuso de la selva. Los recursos del trópico húmedo. Información Científica y Tecnológica :3538.

Guariguata M.R., Chazdon R.L., Denslow J.S., Dupuy J.M. y Anderson L. 1997. Structure and floristic of secondary and oldgrowth stands in lowland Costa Rica. Plant Ecology:107-120.

Guariguata M.R. y Ostertag R. 2002. Sucesión secundaria. En: Guariguata M.R. y Kattan G.H. Eds. Ecología y Conservación de Bosques Neotropicales, pp. 591-623, Libro Universitario Regional, Cartago.

Guariguata M.R. y Ostertag R. 2001. Neotropical secondary forest succession: changes in structure and functional characteristics. Forest Ecology and Management :185-206.

Herrerías-Diego Y. y Benítez-Malvido J. 2005. Consecuencias de la fragmentación de ecosistemas. En: Sánchez O., Peters E., Márquez-Huitzil R., Vega E., Portales G., Valdéz M. y Azuara D. Eds. Temas sobre la Restauración Ecológica, pp. 113-126, Secretaría de Medio Ambiente y Recursos Naturales-Instituto Nacional de Ecología, U.S. Fish \& Wildlife Services-Unidos para la Conservación A.C., México, D.F.

INEGI [Instituto Nacional de Estadística y Geografía]. 1985a. Carta geológica. Calkiní F15-9-12. Escala 1:250 000, México, D.F.

INEGI. 1985b. Carta edafológica. Calkiní F15-9-12. Escala 1:250 000, México, D.F.

Lavin M., Herendeen P.S. y Wojciechowski M.F. 2005. Evolutionary rates analysis of Leguminosae implicates a rapid diversification of lineages during the Tertiary. Systematic Biology :575-594.

Lebrija-Trejos E., Brongers F., Pérez-García E.A y Meave J.A. 2008. Successional change and resilience of a very dry tropical deciduous forest following shifting agriculture. Biotropica :422-431.

Lott E.J., Bullock S.H. y Solís-Magallanes J.A. 1987. Floristic diversity and structure of Upland and Arroyo Forest of Coastal Jalisco. Biotropica :228-235.

Magurran A.E. 1988. Ecological Diversity and its Measurement. Princeton University Press, Princeton.

Marín-Chávez J. 1997. Composición y estructura de la vegetación y sus relaciones con el banco de semillas en tres sitios de la selva baja caducifolia de la Península de Yucatán. Tesis de Maestría. Facultad de Medicina Veterinaria y Zootecnia, Universidad Autónoma de Yucatán, Mérida, 105 pp.

Martínez E. y Galindo-Leal C. 2002. La vegetación de Calakmul, Campeche, México: clasificación, descripción y distribución. Boletín de la Sociedad Botánica de México :7-32.

Martínez-Ramos M. y García-Orth X. 2007. Sucesión ecológica y restauración de las selvas húmedas. Boletín de la Sociedad Botánica de México :69-84.

Miranda F. 1978. Vegetación de la Península Yucateca. Colegio de Postgraduados-Secretaría de Agricultura y Recursos Hidráulicos, Chapingo.

Miranda F. y Hernández-X. E. 1963. Los tipos de vegetación de México y su clasificación. Boletín de la Sociedad Botánica de 
México :29-179.

Mizrahi A., Ramos-Prado J.M. y Jiménez-Osornio J. 1997. Composition, structure and management potential of secondary dry tropical vegetation in two abandoned plantations of Yucatan, Mexico. Forest Ecology and Management :273-282.

Montenegro-S. A.L., Ávila-Parra Y.A., Mendivelso-Ch H.A. y Vargas O. 2006. Potencial del banco de semillas en la regeneración de la vegetación del humedal Jaboque, Bogotá, Colombia. Caldasia :285-306.

Mueller-Dombois D. y Ellenberg H. 2002. Aims and Methods of Vegetation Ecology. The Blackburn Press, Nueva York.

Orians G.H. 2000. Biodiversity and ecosystem processes in tropical ecosystems. Revista de Biología Tropical :297-303.

Peña-Claros M. 2003. Changes in forest structure and species composition during secondary forest succession in the Bolivian Amazon. Biotropica :450-461.

Pineda-García F., Arredondo-Amezcua L. e Ibarra-Manríquez G. 2007. Riqueza y diversidad de especies leñosas del bosque tropical caducifolio El Tarimo, Cuenca del Balsas, Guerrero. Revista Mexicana de Biodiversidad:129-139.

Pennington T.D. y Sarukhán J. 2005. Árboles Tropicales de México. Manual para la Identificación de las Principales Especies. Universidad Nacional Autónoma de México-Fondo de Cultura Económica, México, D.F.

Ramírez-Marcial N., Ochoa-Gaona S., González-Espinosa M. y Quintana-Ascencio P.F. 1998. Análisis florístico y sucesional en la estación biológica Cerro Huitepec, Chiapas, México. Acta Botanica Mexicana :59-85.

Rico-Gray V. 1982. Estudio de la vegetación de la zona costera inundable del noroeste del estado de Campeche, México: Los Petenes. Biótica :171-190.

Rico-Gray V. y García-Franco J.G. 1991. The maya and the vegetation of the Yucatan Peninsula. Journal of Ethnobiology :135142.

Rico-Gray V. y García-Franco J.G. 1992. Vegetation and soil seed bank of successional stages in tropical lowland deciduous forest. Journal of Vegetation Science :617-624.

Rico-Gray V., García-Franco J.G., Puch A. y Sima P. 1988. Composition and structure of a tropical dry forest in Yucatan, Mexico. International Journal Ecology Environmental Science :2129.

Recibido: 29 de octubre de 2010

Aceptado: 24 de agosto de 2011
Ruprecht E. 2005. Secondary succession in old-fields in the Transylvanian Lowland (Romania). Preslia, Praha:145-157.

Rzedowski J. 1978. Vegetación de México. Limusa, México, D.F.

Sánchez-Aguilar R.L. y Rebollar-Domínguez S. 1999. Deforestación en la Península de Yucatán, los retos que enfrenta. Madera y Bosques :3-17.

Sagar R. y Singh J.S. 2005. Structure, diversity, and regeneration of tropical dry deciduous forest of northern India. Biodiversity and Conservation :935-959.

Segura G., Balvanera P., Durán E. y Pérez A. 2003. Tree community structure and stem mortality along a water availability gradient in a Mexican tropical dry forest. Plant Ecology :259-271.

Sousa-S. M. y Delgado-S. A. 1998. Leguminosas mexicanas: fitogeografía, endemismo y orígenes. In: Ramamoorthy T.P., Bye R., Lot A. y Fa J. Eds. Diversidad Biológica de México: Orígenes y Distribución, pp. 449-500, Universidad Nacional Autónoma de México, México, D.F.

Thompson J., Brokaw N., Zimmerman J.K., Waide R.B., Everham III E.M., Lodge D.J., Taylor C.M., García-Montiel D. y Fluet M. 2002. Land use history, environment, and tree composition in a tropical forest. Ecological Applications :1344-1363.

Toledo M. y Salick J. 2006. Secondary succession and indigenous management in semideciduous forest fallows of the Amazon Basin. Biotropica :161-170.

Trejo I. y Dirzo R. 2000. Deforestation of seasonally dry tropical forest: a national and local analysis in Mexico. Biological Conservation : $133-142$.

Von-Gadow K., Sánchez Orois S. y Aguirre Calderón O.A. 2004. Manejo forestal con bases científicas. Madera y Bosques :316.

Wilson E.O. 1988. The current state of biological diversity. En: Wilson E.O. and Peters F.M. Eds. Biodiversity, pp. 3-18, National Academy Press, Washington, D.C.

Zamora-Crescencio P. 1999. Vegetación y flora de la Unidad de Evaluación y Monitoreo de la Biodiversidad de Hampolol, Campeche, México. Ciencia y el Hombre :27-73.

Zamora-Crescencio P. 2003. Contribución al estudio florístico y descripción de la vegetación del municipio de Tenabo, Campeche, México. Polibotánica :1-40. 
Apéndice. Componentes y valor de importancia de las especies presentes en las parcelas estudiadas en Tepakán, Calkini, Campeche.

\begin{tabular}{|c|c|c|c|c|c|}
\hline Familia & Especie & $\begin{array}{l}\text { Densidad } \\
\text { relativa }\end{array}$ & $\begin{array}{l}\text { Frecuencia } \\
\text { relativa }\end{array}$ & $\begin{array}{l}\text { Dominancia } \\
\text { relativa }\end{array}$ & $\begin{array}{l}\text { Valor de } \\
\text { Importancia }\end{array}$ \\
\hline Boraginaceae & Cordia alliodora (Ruiz \& Pav.) Oken & 15.39 & 8.47 & 10.49 & 34.35 \\
\hline Fabaceae & Piscidia piscipula (L.) Sarg. & 7.57 & 7.63 & 18.72 & 33.91 \\
\hline Fabaceae & Acacia gaumeri S.F.Blake & 15.57 & 7.63 & 10.06 & 33.25 \\
\hline Fabaceae & Lonchocarpus xuul Lundell & 9.30 & 7.20 & 3.88 & 20.38 \\
\hline Fabaceae & Lysiloma latisiliquum (L.) Benth. & 4.70 & 3.81 & 11.28 & 19.79 \\
\hline Sapotaceae & $\begin{array}{l}\text { Sideroxylon obtusifolium } \\
\text { (Roemer \& Schultes) T.D. Penn. }\end{array}$ & 7.57 & 5.08 & 3.96 & 16.61 \\
\hline Fabaceae & Caesalpinia gaumeri Greenm. & 2.35 & 4.24 & 9.48 & 16.06 \\
\hline Fabaceae & Acacia sp. & 3.13 & 4.66 & 3.86 & 11.65 \\
\hline Fabaceae & $\begin{array}{l}\text { Havardia albicans } \\
\text { (Kunth) Britton \& Rose }\end{array}$ & 1.48 & 2.97 & 6.50 & 10.95 \\
\hline Burseraceae & Bursera simaruba (L.) Sarg. & 2.09 & 3.81 & 3.44 & 9.34 \\
\hline Celastraceae & Hippocratea excelsa Kunth & 2.96 & 3.39 & 2.44 & 8.79 \\
\hline Sapotaceae & $\begin{array}{l}\text { Sideroxylon americanum } \\
\text { (Mill.) T.D. Penn. }\end{array}$ & 4.09 & 2.12 & 1.76 & 7.97 \\
\hline Ebenaceae & Diospyros anisandra S.F. Blake & 2.96 & 3.39 & 0.84 & 7.19 \\
\hline Rhamnaceae & Colubrina sp. & 2.78 & 0.85 & 1.43 & 5.06 \\
\hline Rubiaceae & Guettarda elliptica Sw. & 1.57 & 2.97 & 0.49 & 5.02 \\
\hline Ebenaceae & Diospyros cuneata Standl. & 1.48 & 2.97 & 0.45 & 4.89 \\
\hline Erythroxylaceae & Erythroxylum rotundifolium Lunan & 1.65 & 1.69 & 1.42 & 4.77 \\
\hline Nyctaginaceae & Neea choriophylla Standl. & 1.13 & 2.12 & 1.29 & 4.54 \\
\hline Polygonaceae & Gymnopodium floribundum Rolfe & 1.65 & 1.27 & 1.23 & 4.15 \\
\hline Fabaceae & Diphysa carthagenensis Jacq. & 1.13 & 2.12 & 0.69 & 3.94 \\
\hline Sapindaceae & Thouinia paucidentata Radlk. & 1.04 & 2.12 & 0.55 & 3.71 \\
\hline Fabaceae & Arbusto (Fabaceae) & 0.52 & 1.27 & 1.70 & 3.49 \\
\hline Malvaceae & Helicteres baruensis Jacq. & 0.70 & 2.54 & 0.21 & 3.45 \\
\hline Fabaceae & Lonchocarpus rugosus Benth. & 1.13 & 1.27 & 0.64 & 3.04 \\
\hline Ebenaceae & Diospyros verae-crucis (Standl.) Standl. & 0.61 & 1.69 & 0.22 & 2.53 \\
\hline Euphorbiaceae & $\begin{array}{l}\text { Cnidoscolus multilobus } \\
\text { (Pax.) I.M. Johnston }\end{array}$ & 0.35 & 1.69 & 0.29 & 2.33 \\
\hline Brassicaceae & Capparis indica (L.) Druce & 0.43 & 1.69 & 0.20 & 2.33 \\
\hline Boraginaceae & Cordia sp. & 0.61 & 0.85 & 0.63 & 2.08 \\
\hline Anacardiaceae & Spondias sp. & 0.78 & 0.85 & 0.24 & 1.87 \\
\hline Fabaceae & $\begin{array}{l}\text { Pithecellobium lanceolatum (Humb. \& } \\
\text { Bonpl. Ex Willd.) Benth. }\end{array}$ & 0.52 & 0.85 & 0.44 & 1.81 \\
\hline Malpighiaceae & Malpighia glabra L. & 0.43 & 0.85 & 0.19 & 1.47 \\
\hline Fabaceae & Mimosa bahamensis Benth. & 0.26 & 0.85 & 0.13 & 1.24 \\
\hline Myrtaceae & Eugenia sp. & 0.52 & 0.42 & 0.16 & 1.11 \\
\hline Fabaceae & Bauhinia divaricata L. & 0.17 & 0.85 & 0.03 & 1.06 \\
\hline
\end{tabular}


Apéndice. Continuación

\begin{tabular}{|c|c|c|c|c|c|}
\hline Familia & Especie & $\begin{array}{l}\text { Densidad } \\
\text { relativa }\end{array}$ & $\begin{array}{l}\text { Frecuencia } \\
\text { relativa }\end{array}$ & $\begin{array}{l}\text { Dominancia } \\
\text { relativa }\end{array}$ & $\begin{array}{c}\text { Valor de } \\
\text { Importancia }\end{array}$ \\
\hline Polygonaceae & $\begin{array}{l}\text { Neomillspaughia emarginata } \\
\text { (H. Gross) Blake }\end{array}$ & 0.26 & 0.42 & 0.29 & 0.98 \\
\hline Bignoniaceae & Parmentiera sp. & 0.26 & 0.42 & 0.05 & 0.74 \\
\hline Salicaceae & Xylosma sp. & 0.26 & 0.42 & 0.05 & 0.74 \\
\hline Nyctaginaceae & Pisonia aculeata L. & 0.17 & 0.42 & 0.03 & 0.63 \\
\hline Myrtaceae & Psidium sp. & 0.09 & 0.42 & 0.11 & 0.62 \\
\hline Fabaceae & Acacia cornigera (L.) Willd. & 0.09 & 0.42 & 0.05 & 0.56 \\
\hline Rhamnaceae & $\begin{array}{l}\text { Karwinskia humboldtiana (Willd. ex } \\
\text { Roem. \& Schult.) Zucc. }\end{array}$ & 0.09 & 0.42 & 0.05 & 0.56 \\
\hline Rubiaceae & Randia longiloba Hemsl. & 0.09 & 0.42 & 0.03 & 0.54 \\
\hline \multirow[t]{2}{*}{ Theophrastaceae } & Jacquinia sp. & 0.09 & 0.42 & 0.02 & 0.53 \\
\hline & & 100 & 100 & 100 & 300 \\
\hline
\end{tabular}

